ARTICLE

\title{
High-resolution imaging and computational analysis of haematopoietic cell dynamics in vivo
}

Claire S. Koechlein ${ }^{1,2, \star}$, Jeffrey R. Harris ${ }^{3, \star}$, Timothy K. Lee ${ }^{4}$, Joi Weeks ${ }^{1,2}$, Raymond G. Fox ${ }^{1,2}$, Bryan Zimdahl ${ }^{1,2,3}$, Takahiro Ito ${ }^{1,2,3}$, Allen Blevins ${ }^{1,2}$, Seung-Hye Jung ${ }^{3}$, John P. Chute ${ }^{5,6}$, Amit Chourasia ${ }^{7}$, Markus W. Covert ${ }^{4} \&$ Tannishtha Reya $a^{1,2,3}$

Although we know a great deal about the phenotype and function of haematopoietic stem/progenitor cells, a major challenge has been mapping their dynamic behaviour within living systems. Here we describe a strategy to image cells in vivo with high spatial and temporal resolution, and quantify their interactions using a high-throughput computational approach. Using these tools, and a new Msi2 reporter model, we show that haematopoietic stem/progenitor cells display preferential spatial affinity for contacting the vascular niche, and a temporal affinity for making stable associations with these cells. These preferences are markedly diminished as cells mature, suggesting that programs that control differentiation state are key determinants of spatiotemporal behaviour, and thus dictate the signals a cell receives from specific microenvironmental domains. These collectively demonstrate that high-resolution imaging coupled with computational analysis can provide new biological insight, and may in the long term enable creation of a dynamic atlas of cells within their native microenvironment.

\footnotetext{
${ }^{1}$ Departments of Pharmacology and Medicine, School of Medicine, University of California, San Diego, La Jolla, California 92093, USA. ${ }^{2}$ Sanford Consortium for Regenerative Medicine, La Jolla, California 92037, USA. ${ }^{3}$ Department of Pharmacology and Cancer Biology, Duke University Medical Center, Durham, North Carolina 27710, USA. ${ }^{4}$ Department of Bioengineering, Stanford University, Stanford, California 94305, USA. ${ }^{5}$ Division of Cellular Therapy, Duke University Medical Center, Durham, North Carolina 27710, USA. ${ }^{6}$ Division of Hematology Oncology, Department of Medicine, University of California, Los Angeles, Los Angeles, California 90095, USA. ${ }^{7}$ San Diego Supercomputer Center, University of California, San Diego, La Jolla, California 92093, USA. * These authors contributed equally to this work. Correspondence and requests for materials should be addressed to M.C. (email: mcovert@stanford.edu) or to T.R. (email: treya@ucsd.edu).
} 
T he haematopoietic system is responsible for generating all the cells of the blood and immune system. The development of fully mature cells from immature haematopoietic stem and progenitor cells occurs in a highly regulated manner within the bone marrow, the primary site of adult haematopoiesis ${ }^{1}$. Here cells integrate a multitude of soluble and cell contact-derived signals from their microenvironment or niche to achieve and maintain tissue homeostasis ${ }^{2-4}$, as well as to initiate regeneration in response to injury ${ }^{5}$. Defining the dynamic interactions of haematopoietic cells with the microenvironment over time and space is thus critically important to better understanding haematopoiesis.

Traditionally, studies of these interactions have been largely restricted to static analysis primarily due to limitations in imaging technology and tissue accessibility ${ }^{6-12}$. Of note, advances in the field have improved the utility of this approach. For example, in a recent study, optical clearing of the bone marrow permitted deep confocal imaging of haematopoietic cells and digital reconstruction of the marrow cavity ${ }^{13}$. However, the dynamic changes that occur as cells interact with components of the bone marrow microenvironment are not readily captured by these methods. To address this, several groups have used two-photon intravital imaging within the bone marrow cavity of the calvarium ${ }^{14-16}$ or the long bone ${ }^{17}$. While these studies have provided valuable new ways to visualize the haematopoietic compartment and to generate three-dimensional spatial models of the bone marrow microenvironment in living animals, there is a continued need for not only increasing spatiotemporal resolution but also a strategy to track endogenous cells without transplantation and a means by which the 'big data' that is generated by such imaging approaches can be analysed to reveal new biological patterns. This would enable us to better map the interactions, signals and mechanisms that govern haematopoietic cell behaviour and function in vivo, and thereby understand how this can fail in disease and degeneration.

To address this need, we have developed an approach that allows real-time imaging of haematopoietic cells in the context of their living microenvironment with high spatial and temporal resolution. Notably, the resolution achieved has allowed us to build a new analytic tool that permits in vivo tracking of individual cells and their temporal and spatial behaviour relative to microenvironmental niches. In addition to tracking transplanted haematopoietic cells, we also tracked endogenous immature haematopoietic cells using a newly developed Musashi2 (Msi2) knock-in reporter mouse. This mouse reports endogenous expression of Musashi2 (reporter for Musashi2, REM2) with enhanced green fluorescence protein (eGFP) ${ }^{18}$. Because Msi2 is highly expressed within haematopoietic stem and progenitor cells $^{19}$, Msi2GFP bright expression faithfully marks an immature haematopoietic population, which can be dynamically tracked in vivo. This reporter mouse, in conjunction with high-resolution live imaging, makes it possible to dynamically track endogenous immature cells in vivo. Using these tools, we have identified spatial 'hotspots' within the microenvironment: areas where haematopoietic stem and progenitor cells preferentially reside and interact. Specifically, we find that immature haematopoietic cells have a significant preference for being in stable contact with vascular domains, while differentiated cells make more short-term interactions and frequently shuttle between the vascular and endosteal domains. These suggest that differentiation state can control the spatiotemporal behaviour of haematopoietic cells and the programs that control cell fate also dictate the kinds of signals cells will be exposed to by the virtue of their localization in specific microdomains. These data show that high-resolution imaging coupled with an effective high-throughput computational approach can provide new biological insight into the dynamics of haematopoietic cells in their microenvironment, and can be used to establish a baseline to study the changes in haematopoietic cell interactions within the niche during regeneration and oncogenesis.

\section{Results}

Real-time imaging. To understand how haematopoietic stem and progenitor cells behave in living tissues, we developed a real-time imaging strategy to visualize cells in high-resolution over extended periods of time. We used fluorescent protein-expressing transgenic mice to observe the spatial orientation of the bone marrow cavity, and a typical confocal microscope to view inside the mouse calvarium (Fig. 1a) ${ }^{20}$. Mice were anaesthetized, their calvaria exposed and they were placed either inverted on an imaging apparatus or upright in a stereotactic device. Stabilization of animals was important for limiting breathing artefacts that can occur during an imaging session, and consistency of animal orientation was crucial for successful imaging of the same region (in the parasagittal sinusoids) between mice and between experiments over extended periods of time (Supplementary Fig. 1).

To highlight the features of the bone marrow microenvironment, mice with constitutive expression of dsRed under the control of a ubiquitous promoter were used ${ }^{21}$. This strategy provided a counterlabel and had a 'backlighting' effect for visualizing microenvironment cells. A typical $\times 10$ image of the bone marrow of a dsRed mouse is shown in Fig. 1b. When analysing haematopoietic cell movement, we used a higher magnification objective $(\times 20)$ to achieve greater spatial resolution (Fig. 1c). The use of transgenic mice expressing dsRed.T3 (ref. 22) to create a labelled microenvironment was particularly important in allowing tracking of haematopoietic cell encounters and associations with the niche at a single-cell level.

The high degree of temporal and spatial resolution allowed clear visualization of transplanted cells interacting for several minutes to several hours with specific niches. In these experiments, actin promoter-driven $\mathrm{GFP}^{+}$haematopoietic progenitor cells, as defined by the absence of lineage markers (lineage negative or $\operatorname{Lin}^{-}$), were transplanted into dsRed recipient mice (Fig. 1d; Supplementary Movie 1). This strategy provided a way to distinguish associations that lasted short periods of time and those that were more stable, lasting several hours. Further, individual $\mathrm{GFP}^{+}$cells could be tracked rolling/crawling along the endothelium within the calvarial marrow (Fig. 1d, arrows; Supplementary Movie 2). Finally, we were able to trace individual cells dividing in real time (Fig. 1e; Supplementary Movie 3), a testament to the single-cell resolution achieved in this system.

Different fluorescent proteins with multiple spectra were used to analyse distinct cell populations simultaneously. To test imaging in multiple colours, we transplanted bone marrow cells from GFP and cyan fluorescent protein (CFP) donors into dsRed recipients. As shown in the three-dimensional view of the recipient marrow, we could clearly distinguish both $\mathrm{GFP}^{+}$ and $\mathrm{CFP}^{+}$cells within the dsRed backlit microenvironment (Fig. 2a; Supplementary Movie 4). The use of multiple colours enabled comparative imaging of cells from distinct genetic backgrounds within the same niche. We also tested whether we could monitor the signalling status of niche cells. To this end, we crossed actin-dsRed mice to transgenic notch reporter mice (TNR), in which GFP is predominantly expressed in cells responding to the Notch signalling $23-25$. Notch signalling was active in cells surrounding areas of bone and in the endosteal region within the microenvironment (Fig. 2b, asterisks). Further, the association of haematopoietic cells with Notch reporter ${ }^{+}$cells 
a
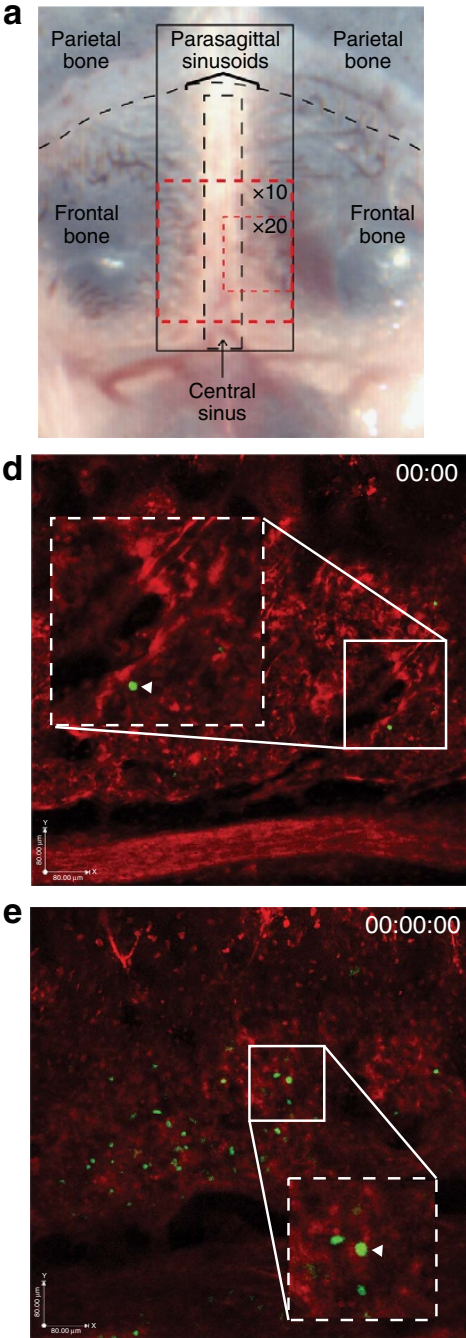

b
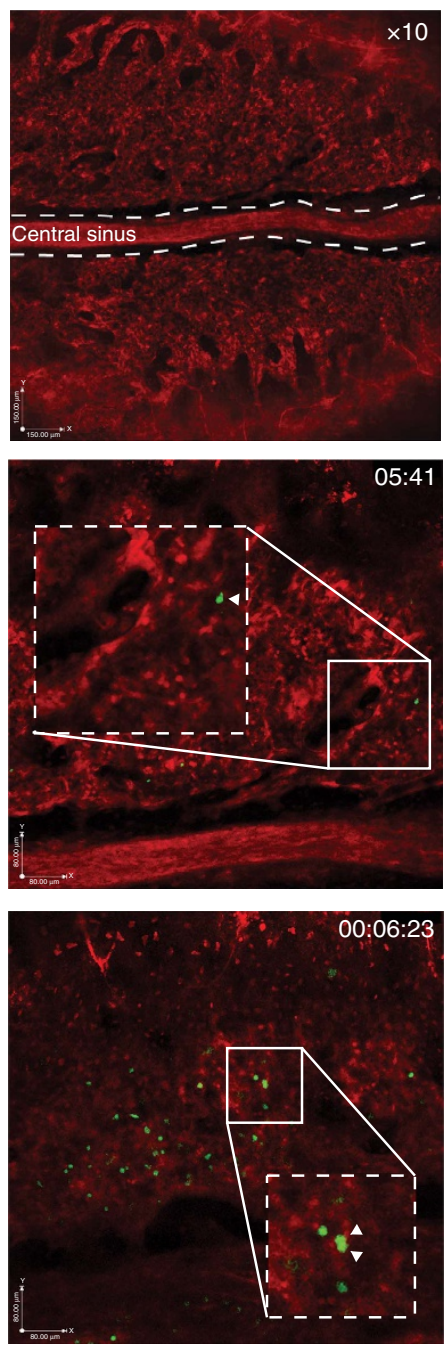
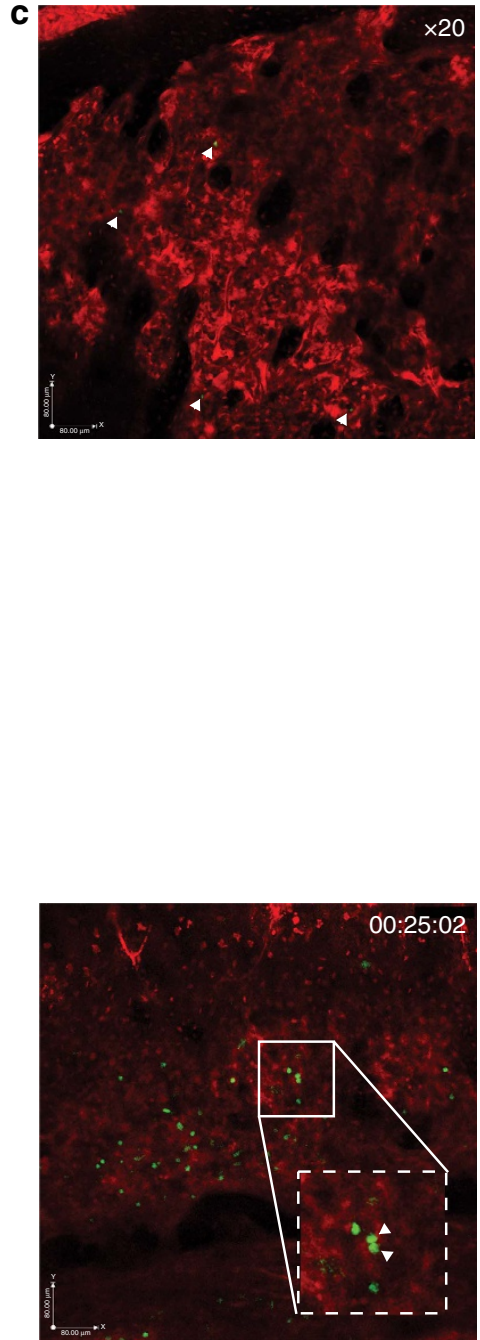

Figure 1 | Real-time imaging. (a) Representative photo showing architecture of imaging area in mouse calvarium. Red-dashed boxes indicate representative areas imaged. Black-dashed box highlights the central sinus. Parasagittal sinusoids flank either side of the central sinus. (b) Representative $\times 10$ image of transgenic dsRed mouse calvarium. White-dashed lines highlight the central sinus. Scale bar, $150 \mu$ m. (c) Representative $\times 20$ image of dsRed bone marrow. Closed triangles depict transplanted Lin - GFP ${ }^{+}$cells in the microenvironment. Scale bar, $80 \mu m$. See Supplementary Movie 1.

(d) Still image of a $\mathrm{Lin}^{-}$GFP + haematopoietic cell (closed triangle) rolling along the vessel wall in a dsRed recipient mouse, shown are images taken at $t=0$ (left panel) and at end point $t=05: 41$ (right panel). See Supplementary Movie 2. Inlay $=\times 1.5$ zoom; scale bar, $80 \mu \mathrm{m}$. (e) Still image of a Lin $-\mathrm{GFP}+$ haematopoietic cell in division. $t=0$ : arrow identifies cell preparing to divide, $t=6: 23$ : arrows indicate cell in the midst of division and $t=25: 02$ : arrows indicate two daughter cells. See Supplementary Movie 3. Scale bar, $80 \mu \mathrm{m}$, box $\times 1.5$ zoom of field.

could be visualized by transplanting dsRed haematopoietic cells into TNR mice crossed with CFP mice (Fig. 2c; Supplementary Movie 5). These data show that haematopoietic cells and their interactions with niche cells responsive to a specific signal can be traced at a single-cell level in vivo.

Computational analysis. The ability to clearly assess niches in real-time coupled with the spatial and temporal resolution allowed us to begin to generate a map of haematopoietic cell associations with the niche in homeostasis. To maintain the most flexibility, we visualized elements of the environment using ectopically delivered antibodies and probes. Vascular endothelial cells and blood vessels were identified using anti-VE-cadherin antibodies and the in vivo probe angiosense, respectively (Fig. 2d; Supplementary Fig. 2a-b; Supplementary Movie 6), and the endosteal region was identified using the in vivo probe OsteoSense (Fig. 2e; Supplementary Fig. 2c-d; Supplementary Movie 7). Other potential niche cells, such as tissue macrophages, could also be visualized using this strategy (Supplementary Fig. 2e; Supplementary Movie 8), and may be of future interest.

The spatial location of $\mathrm{GFP}^{+}$transplanted cells could be clearly viewed relative to the microdomain of interest (Fig. 2d,e, arrows). Beginning with the raw image set, our software automatically corrects for lateral drift between images, identifies individual cells and tracks the position of each cell over time using particle-tracking software (Fig. 3a; Supplementary Movie 9; see Methods). The program then records the $x$ and $y$ coordinates at each time point, as well as the distance travelled and cellular velocity. In addition, with defined endosteal and vascular microdomains (another input to the software), the program calculates the closest distance between these regions and each cell. For example, Fig. 3a shows how one cell, which initially localized close to a vascular (red) region, migrated over time towards an endosteal (grey) region. Figure $3 \mathrm{~b}$ is a trace depicting the quantitative data derived using our software. 
a

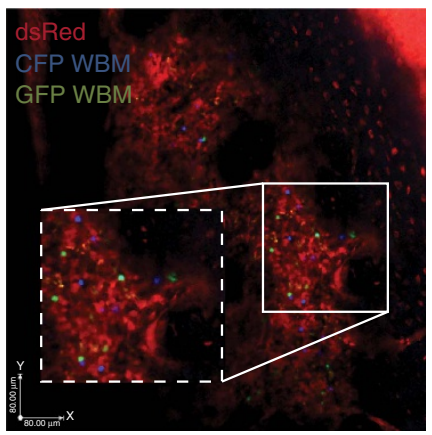

b
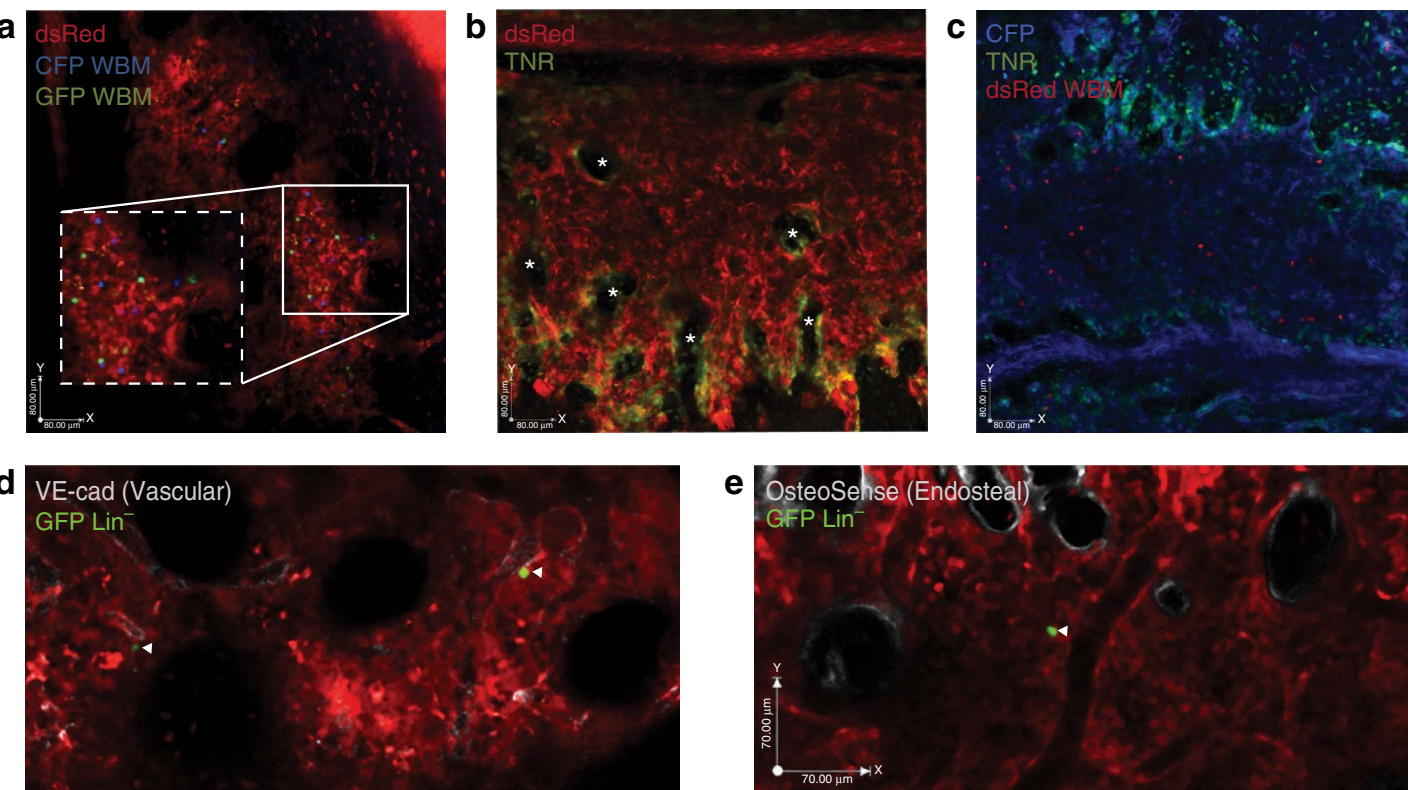

Figure 2 | Multicolour analysis. (a) Representative three-colour analysis of a dsRed recipient transplanted with GFP + and CFP + whole bone marrow (WBM) cells. Corresponding movie shows a z-stack step through the marrow microenvironment (Supplementary Movie 4 ). Scale bar, $80 \mu \mathrm{m}$, box $\times 1.5$ zoom of field. (b) Representative image of dsRed mouse crossed to TNR showing Notch signalling domains within the bone marrow microenvironment; green signal reflects the Notch reporter activity within the microenvironment (asterisks). (c) Representative image of dsRed ${ }^{+}$WBM transplanted into TNR.CFP mouse. Green reflects the Notch reporter activity within the microenvironment (Supplementary Movie 5). Images a-c were obtained

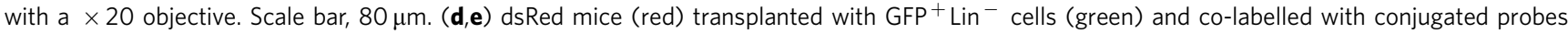
to (d) endothelial cells (anti-VE-cadherin antibody, Supplementary Movie 6) and (e) the endosteal surface (OsteoSense, Supplementary Movie 7). Scale bar, $70 \mu \mathrm{m}$.

Using this approach, we wanted to determine whether there were characteristic distances at which haematopoietic cells interacted with specific regions of the microenvironment. Thus, we first plotted each cell's distance to the vasculature at every time point (Fig. 3c). The resulting histogram suggested that a significant amount of cellular time was spent within $5 \mu \mathrm{m}$ of the vasculature, and, based on visual confirmation, was designated as 'contact'. Interestingly, the region between 5 and $25 \mu \mathrm{m}$ was also highly enriched in terms of cellular presence, and was designated as a 'proximal' zone. Distances greater than $25 \mu \mathrm{m}$ from a niche of interest were designated as a 'distal' zone. These zones held true for the distribution of cells near the endosteum as well (Fig. 3d). These data cumulatively suggested that there are spatial hotspots of associations within the greater haematopoietic microenvironment and allowed us to define the criteria for associations of haematopoietic cells with the niche.

These spatial criteria were then imposed on the trace of the cell tracked in Fig. 3a; this analysis showed that the cell was initially in contact with the vasculature, followed by a proximal interaction with the vasculature and finally a proximal interaction with the endosteal region (Fig. 3e). We added functionality to our software, which enabled us to identify, classify and quantify these interactions automatically (see Methods). Using this tool, we found that the cells we tracked had 95 periods of interaction with either the vascular or endosteal regions and 24 periods of no interaction (that is, classified as distal to both regions) as a group. It is likely that other niche cells within the overall microenvironment can also serve as hotspots for associations and may be intermingled in the 'proximal' and 'distal' zones.

Interestingly, the duration of cell interactions varied from about $4 \mathrm{~min}$ to over $5 \mathrm{~h}$. Of the cells we tracked, $7 \%$ moved through the blood vessels and $12 \%$ moved through the microenvironment in under $2 \mathrm{~min}$ (Fig. 3f); detailed measurements were thus extracted from the rest of the transplanted cells $(n=95)$. On the basis of the distribution of duration of cell interactions, interactions could be categorized into two groups: the cluster of interactions lasting $<60 \mathrm{~min}$ were termed 'short'; and interactions $>60 \mathrm{~min}$ were termed 'long' (Fig. 4a). In the cellular trace used as an example in Fig. 4b, imposing such temporal criteria shows that the cell tracked in this case made one short contact with the vasculature lasting $<30 \mathrm{~min}$ and remained distal to the endosteal niche at all times.

Comparative dynamics. The ability to define interactions in terms of space and time provided suitable metrics for quantitatively comparing interactions made by distinct groups of cells. Using this approach, we compared the dynamics of transplanted haematopoietic cells at different stages of differentiation in a normal environment. Specifically, we compared the behaviour of three cell populations: (1) a stem

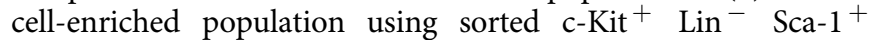
(KLS), (2) a progenitor cell-enriched population using a lineage depletion ( $\left.\mathrm{Lin}^{-}\right)$and (3) a fully differentiated lineage-positive fraction $\left(\mathrm{Lin}^{+}\right)$isolated from the bone marrow. This comparison revealed marked temporal and spatial differences in the interactions of mature and immature haematopoietic cells with their microenvironment. As shown in Fig. 4c, KLS cells made approximately threefold more long interactions per cell with the vascular niche than Lin $^{-}$progenitors cells, which made mostly short associations. In contrast, both $\mathrm{Lin}^{-}$progenitors and KLS cells made more short-term interactions with the endosteal niche than they did long-term associations (Fig. 4d). Moreover, haematopoietic cells were found to associate with vascular niches the majority of the time (Fig. 4e) and, consistent with their 
a

Temporal and spatial single cell trace

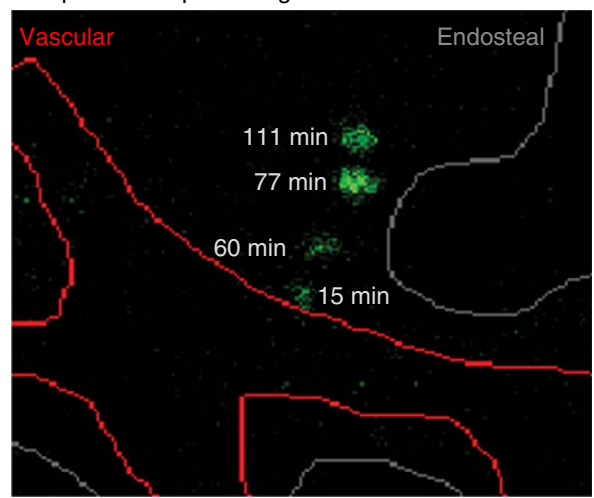

b

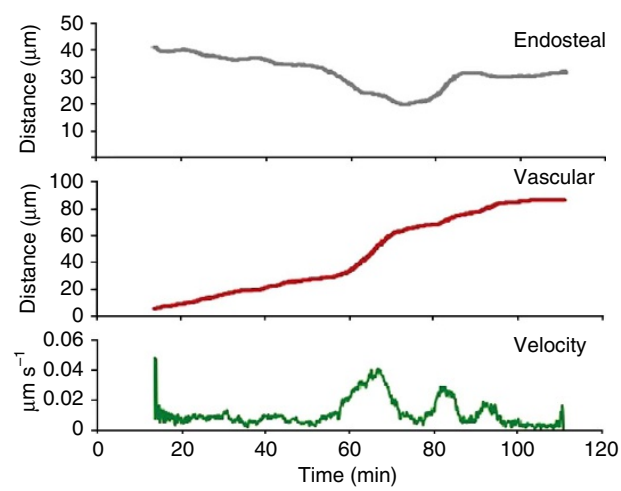

Spatial distribution of haematopoietic cells
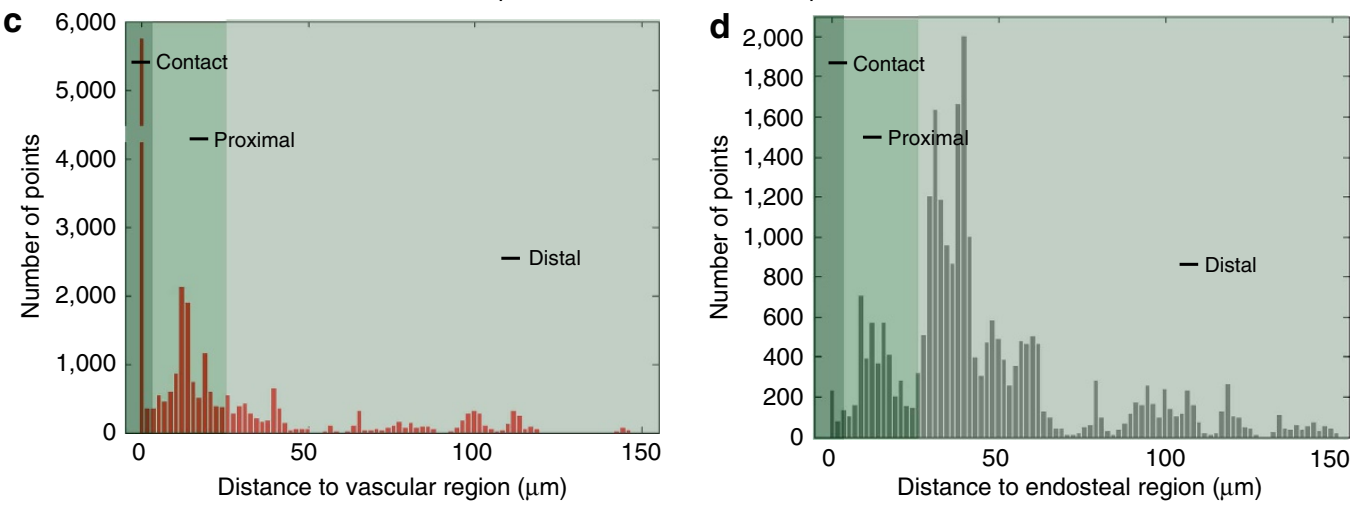

e

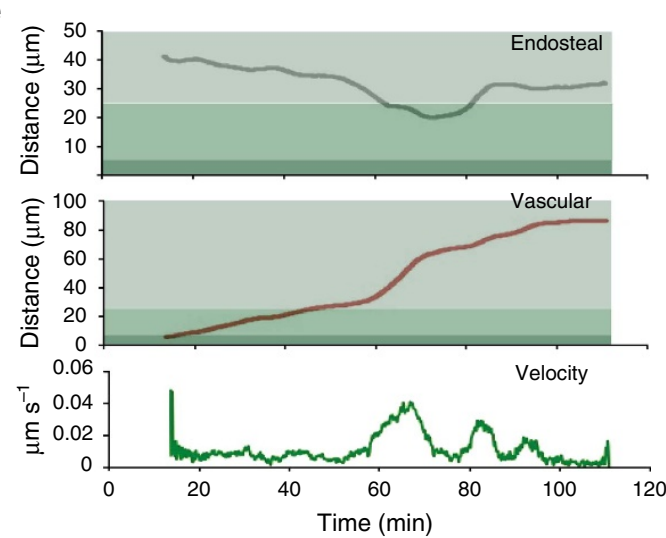

$\mathbf{f}$

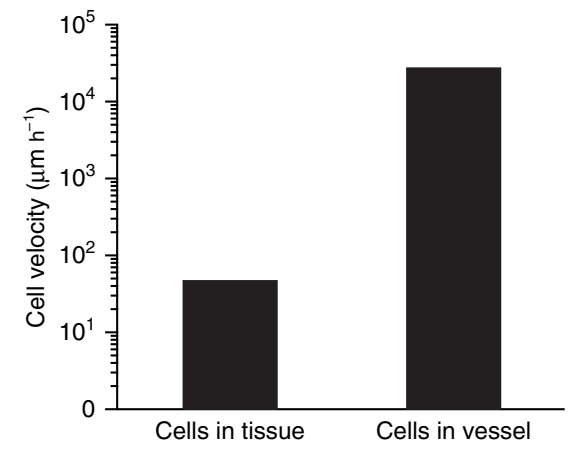

Figure 3 | Computational image analysis of spatial dynamics. (a) Automated analysis enables tracking of individual cells (green) over time, and determination of the distance from the vascular (outlined in red) and endosteal (outlined in grey) regions. See Supplementary Movie 9. (b) For the same cell shown in $\mathbf{a}$, the distance to the endosteal (grey line) and vascular (red line) regions as well as the cell velocity (green line) are shown. (c,d) Histograms containing the distance to the vascular (c) and endosteal (d) regions for all cells analysed at all time points assessed. Three 'zones' were determined from these histograms: contact, proximal and distal. (e) The same data shown in b but with the three zones highlighted. (f) Average cellular velocity for all cells observed in the bone marrow (regardless of lineage status), compared with the average velocity of cells in a blood vessel.

ability to interact with several microenvironmental elements, progenitor cells showed greater displacement from their origin over time (Fig. 4f).

Although the nature of the temporal interactions differed between KLS and $\mathrm{Lin}^{-}$progenitor cells, both of these populations displayed highly significant spatial affinities for contacting the vascular area (Fig. 5a) compared with the endosteum. This preference was greatest in the most undifferentiated cells and decreased with differentiation $(\sim 17$-fold increase in affinity for KLS, 14 -fold for $\mathrm{Lin}^{-}$and 2 -fold for
Lin $^{+}$cells; Fig. 5b). Interestingly, cell interactions were more evenly distributed with the proximal domain of the vascular and endosteal niche (Fig. $5 c, d$ ). These data suggest a model where the programs that control lineage commitment and differentiation are closely linked to the spatial location and temporal interactions of cells within the niche, and that these molecular elements collectively ensure that the most immature cells receive cell-cell contact signals preferentially from the vascular endothelium, and soluble cues from both vascular and endosteal domains (Fig. 5e). 
a
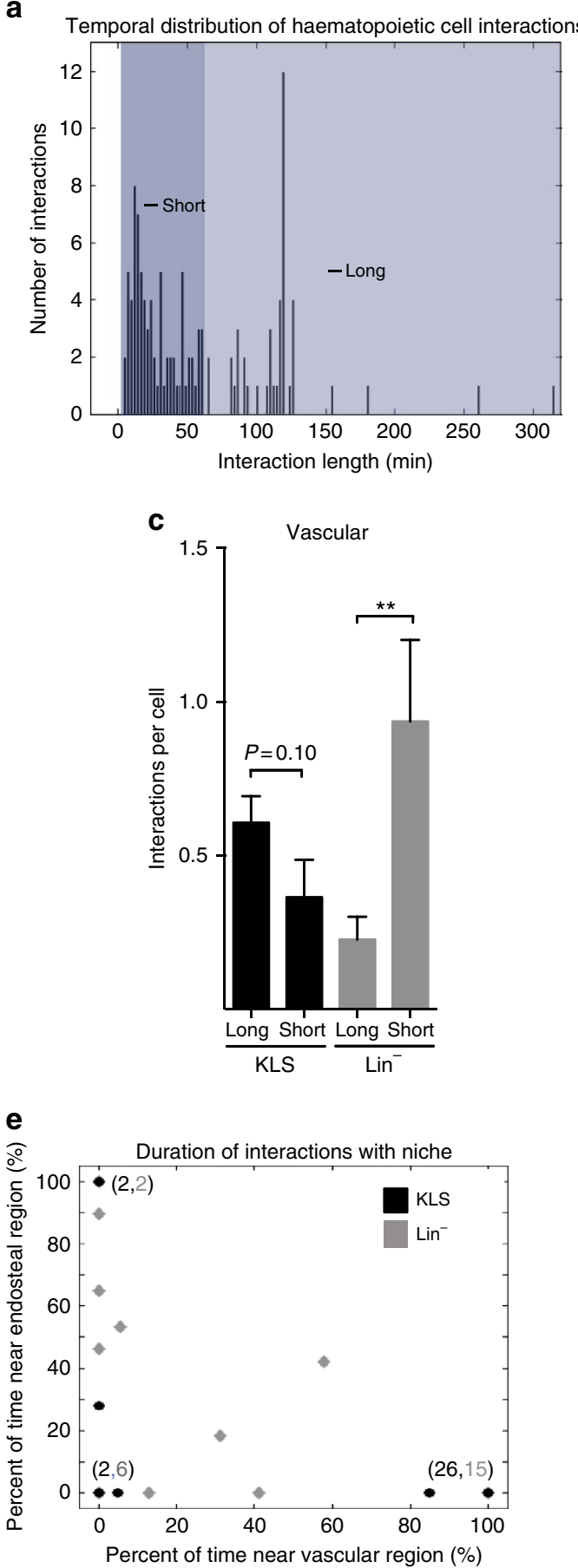

b
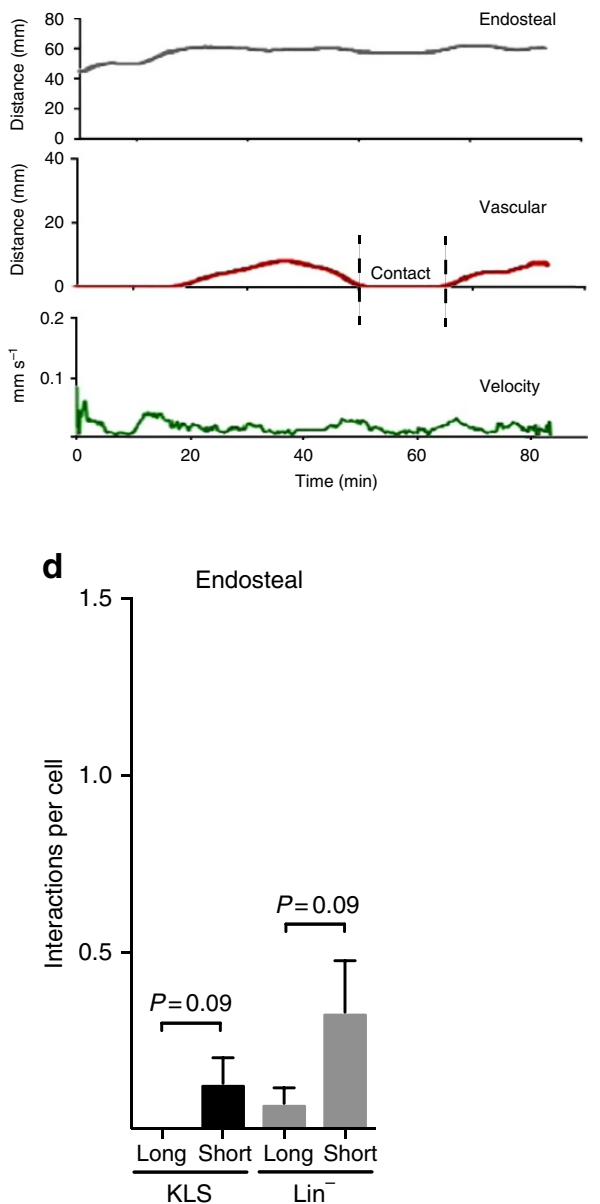

f

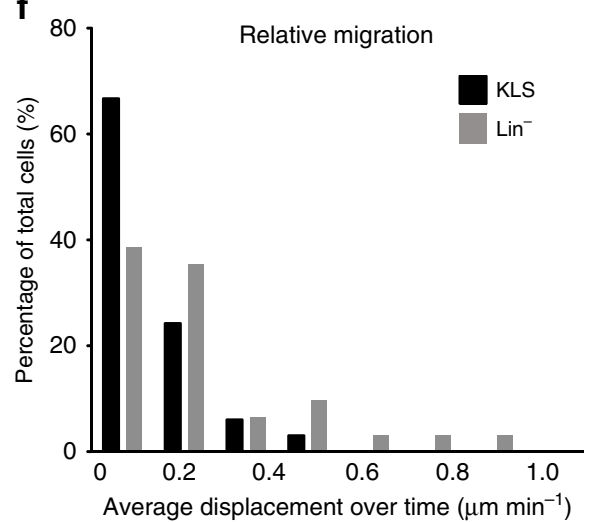

Figure 4 | Comparative temporal dynamics of haematopoietic cells. (a) Histogram showing duration of all the interactions (instances where cells were in contact with or proximal to a given region) calculated by this software. Two categories of interaction duration were identified: short ( $<60$ min) and long (>60 min). (b) Cell trace depicting a short contact with the vascular region. Distance to the endosteal (grey line) and vascular (red line) regions as well as cell velocity (green line) for a representative cell is shown, highlighting a short contact interaction (dashed gates) with the vascular region. (c-f) Categorization of the interactions of KLS (black) and $\mathrm{Lin}^{-}$(grey) cells by region and duration. (c) The incidence of interactions per cell found in the vascular region, sorted by duration. $P=0.10$ for KLS ( $n=33$ cells) and ${ }^{\star \star} P=0.0126$ for $\operatorname{Lin}^{-}(n=31$ cells) by Student's $t$-test. (d) The incidence of interactions per cell found in the endosteal region, sorted by duration. $P=0.09$ for KLS ( $n=33$ cells) and $P=0.09$ for Lin ${ }^{-}$( $n=31$ cells) by Student's $t$-test. Data represented as mean + s.e.m. (e) For each cell, the fraction of observation time (shown as per cent) spent near ( $<25 \mu \mathrm{m}$; in contact or proximal to) the vascular ( $x$ axis) and endosteal ( $y$ axis) regions is plotted as a single point. Point overlap is indicated in the parentheses, where the first number represents KLS (black circle) and the second number Lin ${ }^{-}$(grey diamond) cells that fall in that point. (f) Histogram containing the normalized average displacement for $\mathrm{KLS}_{\text {and }} \mathrm{Lin}^{-}$cells, where displacement is defined as the distance between a current cell position and its first recorded position, and the displacement is normalized by the total time a given cell was observed. 
a

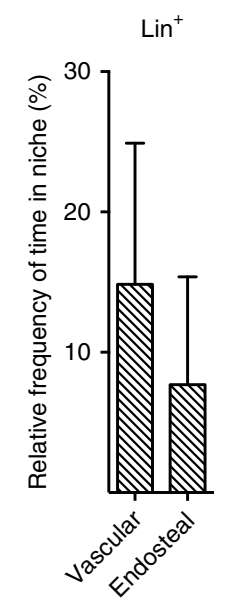

c

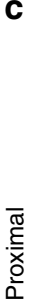

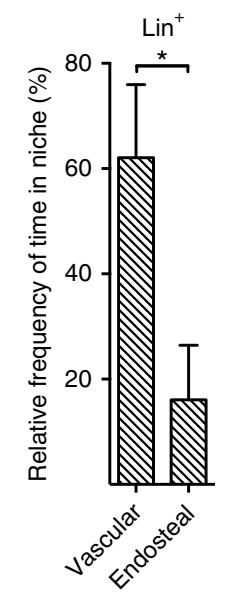

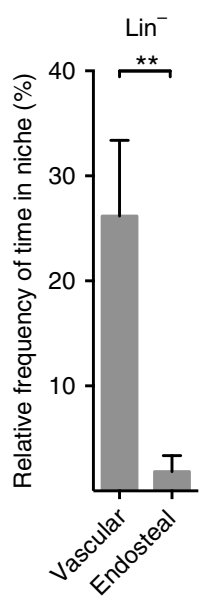

$\operatorname{Lin}^{-}$

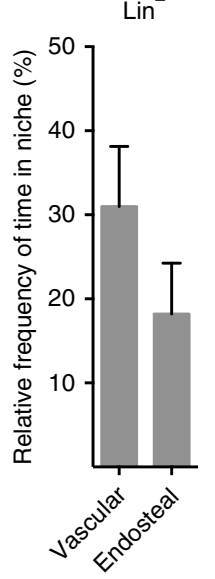

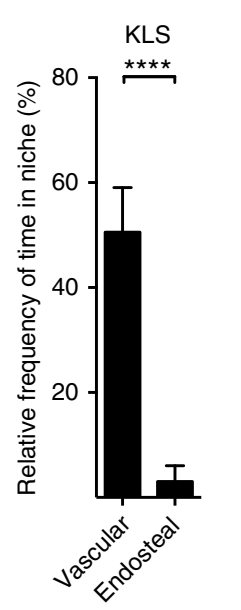

b

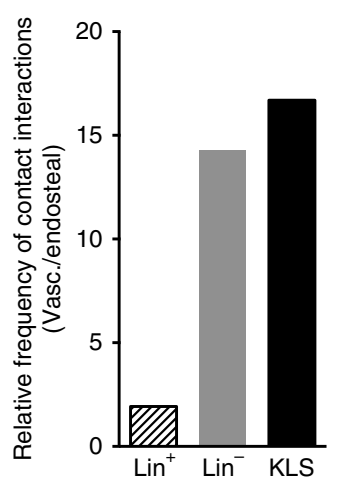

d

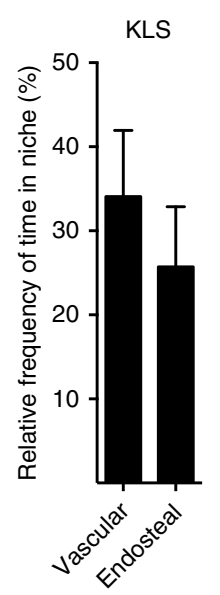

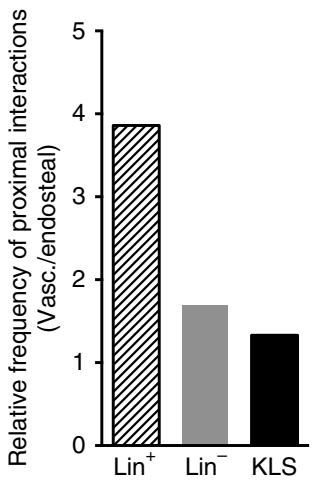

e

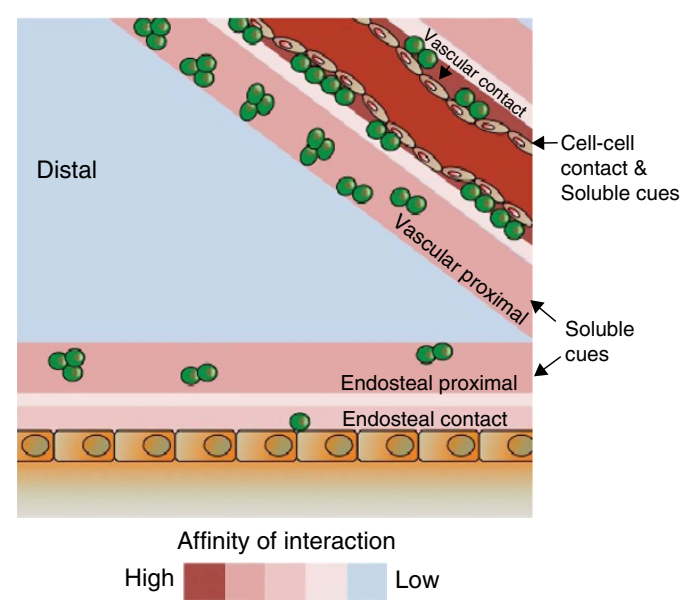

Figure 5 | Comparative associations of haematopoietic cells with vascular and endosteal regions. (a) The relative fraction of time (shown as per cent) in contact with the vascular or endosteal niche. ${ }^{\star \star} P=0.0016$ for $\operatorname{Lin}^{-}{ }^{\star}{ }^{\star \star \star \star} P<0.0001$ for KLS by Student's $t$-test. Data represented as mean + s.e.m. (b) The relative contact frequency (vasculature/endosteal) of KLS (black), Lin ${ }^{-}$(grey) and Lin ${ }^{+}$(solid hatched line) cells. (c) The relative fraction of time in proximal interactions with the vascular or endosteal niche. ${ }^{\star} P=0.0137$ for Lin ${ }^{+}$by Student's $t$-test. Data represented as mean + s.e.m. $(\mathbf{d})$ The relative proximal frequency (vascular/endosteal) of KLS (black), Lin $^{-}$(grey) and Lin ${ }^{+}$(solid hatched line) cells. (e) Model showing microenvironmental regions enriched for progenitor cell $\left(\mathrm{Lin}^{-}\right.$) associations in homeostasis as an example. Cells are preferentially in contact with or proximal to the vascular niche but mostly proximal to, rather than in contact with, the endosteal region. Warmer colours (red) identify areas with a high probability of associations and cooler colours (blue) identify areas with a low probability of associations.

Tracking endogenous haematopoietic cells with an Msi2 reporter. To track endogenous haematopoietic stem and progenitor cells in vivo, we utilized the newly developed REM2 knock-in reporter mouse ${ }^{18}$. This reporter was created by knocking in the eGFP cassette into exon 1 of the Msi2 gene in frame with the ATG start codon. Because this is the first use of the Msi2GFP reporter mouse for imaging normal haematopoietic cells, we wanted to ensure that the disruption of one allele of the 
Msi2 gene caused by insertion of the reporter cassette did not impair stem/progenitor cell function. Our experiments show that $M s i 2^{+/+}$and Msi2 ${ }^{+/ G F P}$ long-term (LT)-HSCs have equivalent colony-forming ability in vitro (Supplementary Fig. 3a) as well as reconstitution ability in vivo (Supplementary Fig. 3b); further we could not detect any difference in the ability of $M s i 2^{+/+}$and Msi2 $2^{+/ G F P}$ stem and progenitor cells to migrate towards chemokines indicating the heterozygous cells likely reflect normal haematopoietic cell behaviour (Supplementary Fig. 3c). In accordance with the known pattern of Msi2 expression, reporter expression was highest in immature stem/progenitor cells and decreased with differentiation and lineage commitment. Specifically, KLS cells, which contain both stem and progenitor cells, and $\mathrm{KLSCD} 0^{+} \mathrm{CD} 48^{-}$cells, which represent highly

\section{a}

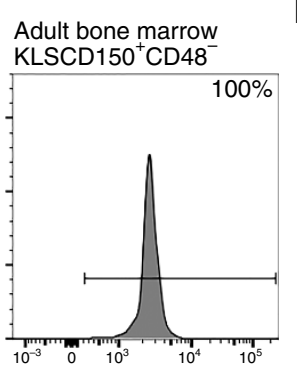

KLS
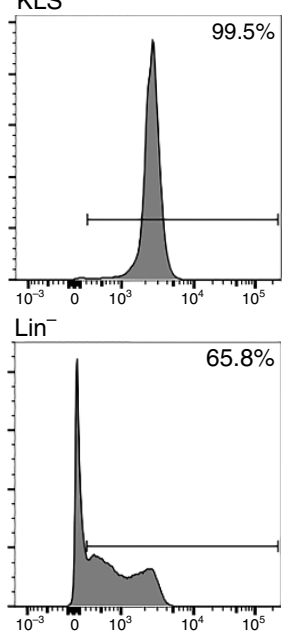

Lin $^{10}$

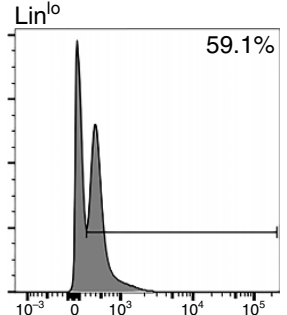

$\operatorname{Lin}^{\text {hi }}$

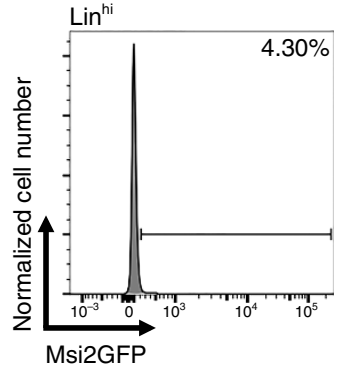

b

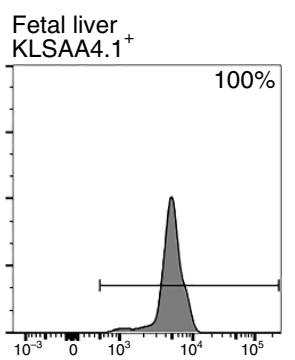

KLS

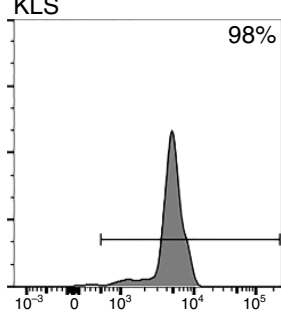

$\operatorname{Lin}^{-}$
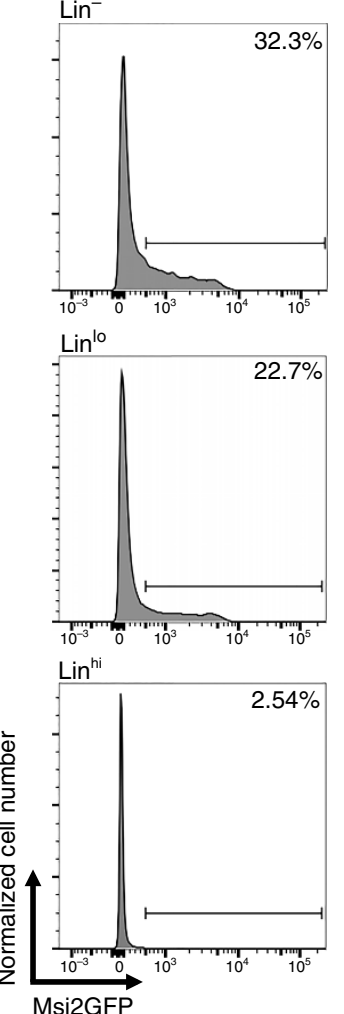
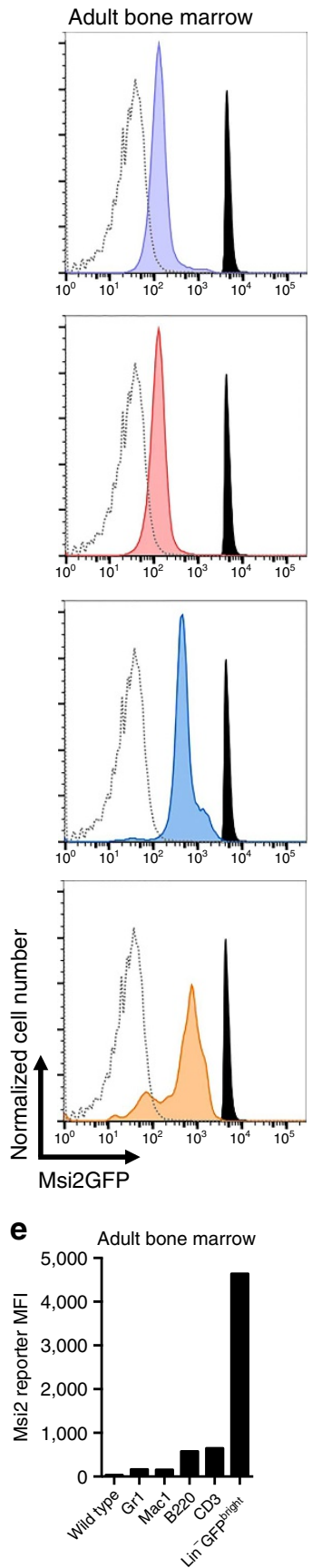

d
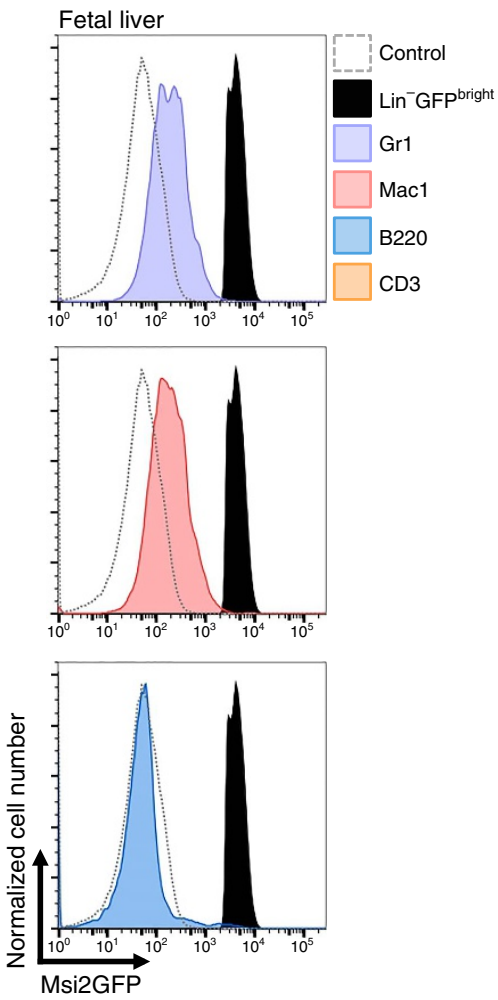

Figure 6 | Msi2 reporter expression in stem/progenitor and differentiated haematopoietic cell populations. (a) Representative flow cytometry plots show histograms of Msi2GFP fluorescence intensity in $\mathrm{KLSCD} 50^{+} \mathrm{CD}_{4}{ }^{-}, \mathrm{KLS}, \mathrm{Lin}^{-}{ }^{-}, \mathrm{Lin}^{\mathrm{lo}}$ and $\mathrm{Lin}^{\text {hi }}$ cells from adult bone marrow. (b) Representative flow cytometry plots show histograms of Msi2GFP fluorescence intensity in KLSAA4.1 ${ }^{+}, \mathrm{KLS}, \mathrm{Lin}^{-}$, Lin ${ }^{10}$ and Lin $^{\text {hi }}$ cells derived from E15.5 fetal liver.

(c) Representative flow cytometry plots showing GFP expression in control (wild type, non-reporter) bone marrow (light grey, dashed), Msi2GFPbright Lincells (black) and differentiated cells from Msi2GFP reporter mouse (coloured). (d) Representative flow cytometry plots showing GFP expression in control (wild type, non-reporter) fetal liver cells (light grey, dashed), Msi2GFPbright Lin ${ }^{-}$cells (black) and differentiated cells from Msi2GFP reporter mouse (coloured). (e) Quantification of mean fluorescence intensity (MFI) of Msi2GFP in control bone marrow (wild type, non-reporter), differentiated lineages and Msi2GFPbright Lin ${ }^{-}$immature cells. (f) Quantification of MFI of Msi2GFP in control fetal liver, differentiated cells and Msi2GFPbright Lin ${ }^{-}$undifferentiated cells. 
purified stem cells, were $99.5 \%$ and $100 \%$ positive for reporter expression, respectively, and contained within the Msi2GFP bright fraction (Fig. 6a). This pattern was also consistent during embryonic development: thus in the fetal liver, KLS cells and the more stem cell pure KLSAA4.1 ${ }^{+}$population were $98 \%$ and $100 \%$ positive for reporter expression, respectively (Fig. 6b), and expression dropped with differentiation. Overall, $\sim 90 \%$ of the Msi2GFP ${ }^{\text {bright }}$ population in the adult bone marrow, and $95 \%$ of the Msi2GFP bright population in the E15.5 fetal liver, were

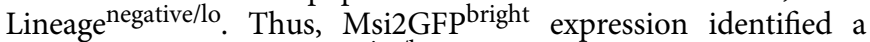

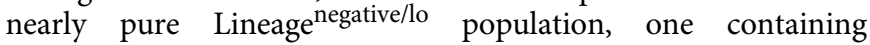
immature uncommitted cells that have not begun to express lineage markers and are thus not lineage committed. While there was some dim Msi2GFP expression in lineage committed cell populations in the bone marrow (B220, CD3, Gr-1 and Mac1), this expression was 7-28-fold dimmer than expression seen in immature cells, consistent with observations in the fetal liver (Fig. 6c-f).

To set up our imaging parameters, we exploited the expression differential between differentiated $\mathrm{B} 220^{+}$cells and Msi2GFP bright cells. Specifically, the fact that $\mathrm{B} 220^{+}$cells were on average eightfold dimmer (Fig. 7a), allowed us to threshold out the Msi2GFPdim cells and visualize only the Msi2GFP bright cells (Fig. 7b,c). The settings used were determined by transplanting Msi2GFP bright and $\mathrm{B}_{22}{ }^{+}$
(Msi2GFP ${ }^{\text {dim }}$ ) cells into separate recipients and defining the voltage gates at which the $\mathrm{B} 220^{+}$cells were undetectable. To facilitate these studies, the Msi2GFP reporter mouse was crossed with a dsRed mouse to provide a counterlabel. Interestingly, all of the Msi2GFP bright cells were localized in contact or proximally to the vasculature, with the majority being in contact with the vascular cells (Fig. $8 \mathrm{a}-\mathrm{c}$ ). In contrast, the majority of Msi2GFP ${ }^{\text {bright }}$ cells observed were localized distally from the endosteum (Fig. 8d-f). In addition to the spatial analysis, we also dynamically tracked Msi2GFP bright cells over time. A representative Msi2GFP ${ }^{\text {bright }}$ cell is shown in contact with the vasculature (Supplementary Fig. 4a). When tracked relative to the vasculature, there was no change in the distribution of the distance over the time tracked (Supplementary Fig. 4b). Consistent with this pattern, none of the Msi2GFP ${ }^{\text {bright }}$ cells moved significantly enough to change the type of association (contact, proximal or distal) they had with the microenvironment. We also examined the spatial interactions of endogenous immature haematopoietic cells within the fetal liver; here Msi2GFP bright cells associate with the vasculature via an even distribution of contact and proximal interactions (Fig. 8g-i). Importantly, these data are consistent with the interactions of transplanted immature cell populations, emphasizing the physiological relevance of our findings that native haematopoietic stem and progenitor cells are particularly dependent on niches set up by vascular domain. a

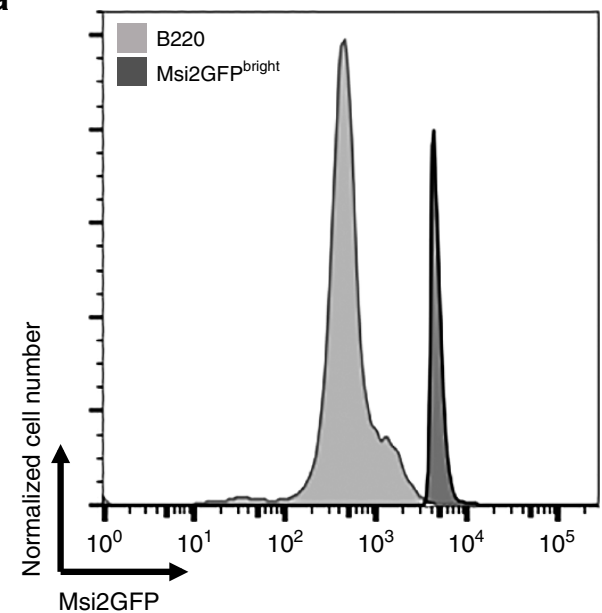

b

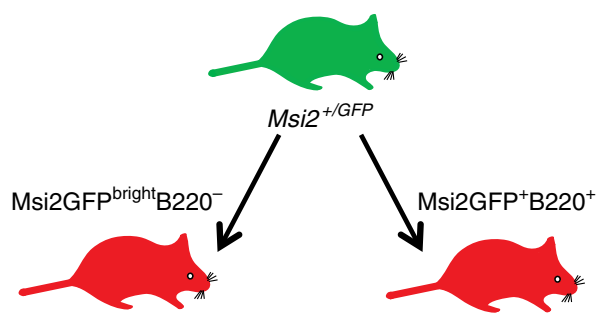

dsRed recipient

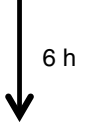

In vivo Imaging

C

Msi2GFP bright $B 220^{-}$

$\left(\mathrm{GFP}^{+}\right)$

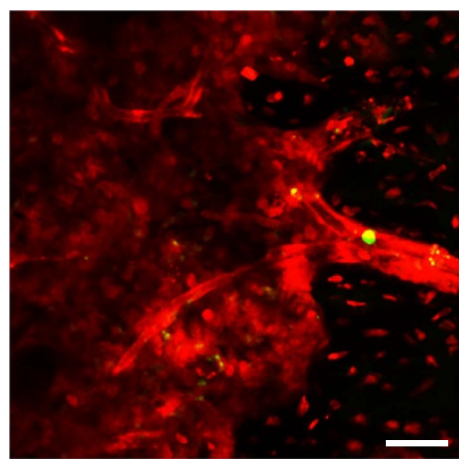

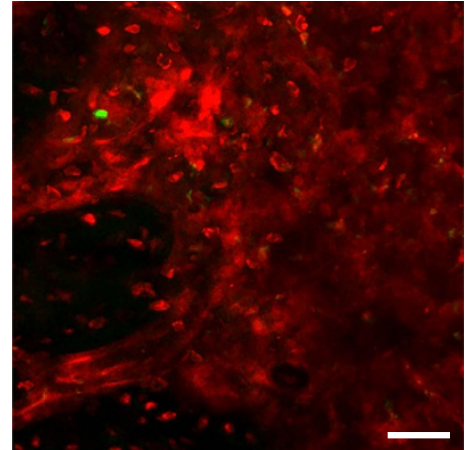
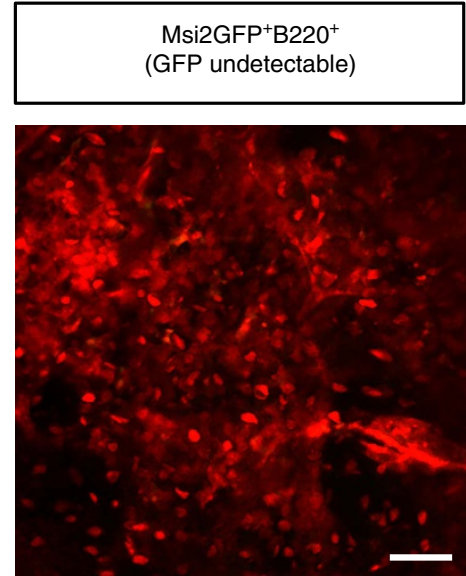

Figure 7 | Imaging Msi2GFPbright cells in vivo enables tracking of endogenous immature cells. (a) Representative flow cytometry plot showing Msi2 reporter fluorescence intensity in $\mathrm{B}_{220^{+}}$cells (light grey) and Msi2GFP ${ }^{\text {bright }}$ cells (dark grey). (b) Experimental design to image and compare fluorescence intensity of Msi2GFP ${ }^{+} \mathrm{B}_{22} \mathrm{O}^{+}$and Msi2GFPbright $\mathrm{B} 22 \mathrm{O}^{-}$cells in vivo. (c) Representative images showing visible transplanted Msi2GFPbright $\mathrm{B} 22 \mathrm{O}^{-}$cells after voltage-gated thresholding so $\mathrm{Msi2GFP}+\mathrm{B}_{22}{ }^{+}$cells were undetectable. Scale bar, $40 \mu \mathrm{m}$. 
a

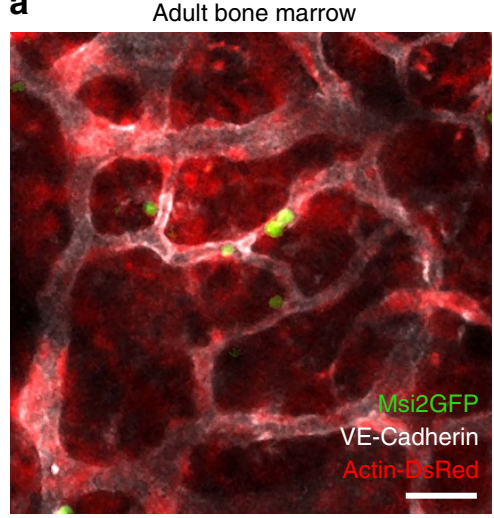

d

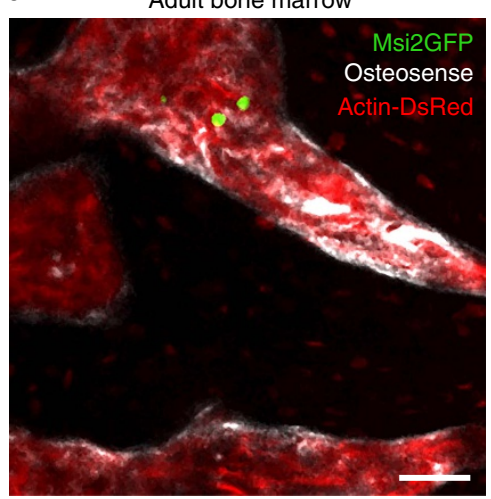

g

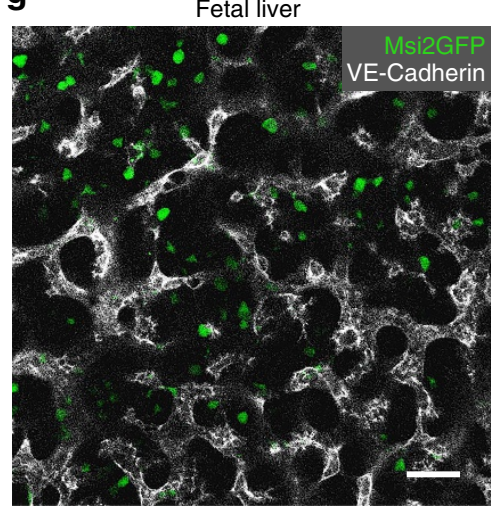

b

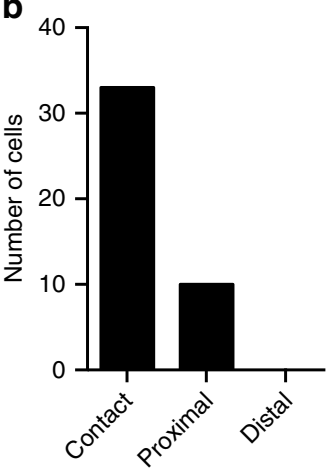

e

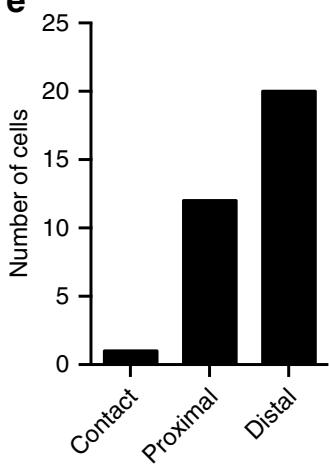

$\mathbf{h}$

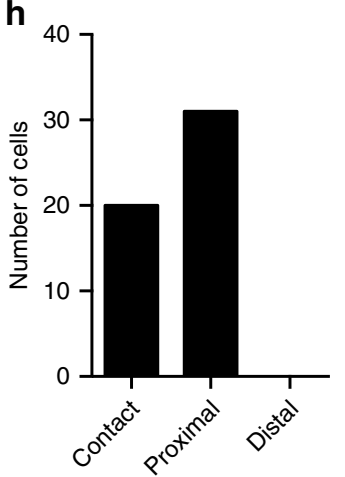

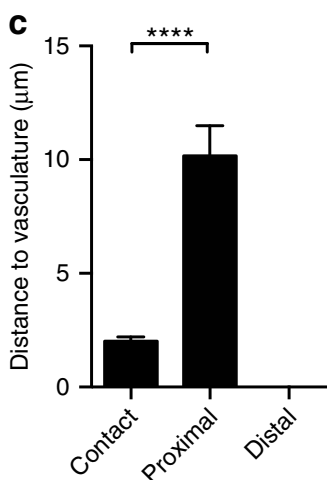
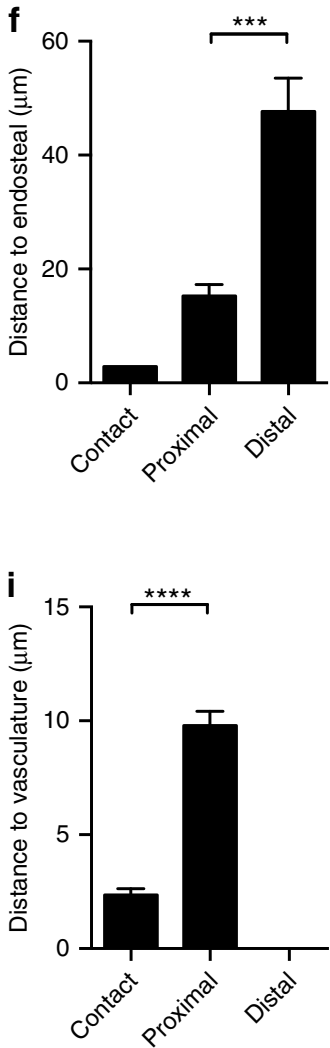

Figure 8 | Comparative associations of endogenous immature haematopoietic cells with vascular and endosteal regions using Msi2 reporter.

Representative image of Msi2GFP (green);dsRed (red) mice showing Msi2GFPbright cells localized near vasculature (a) (white) and endosteum (d) (white). Scale bar, $40 \mu \mathrm{m}$. (b) Quantification showing the incidence of Msi2GFPbright cells localized in contact with, proximal or distal to vasculature ( $n=43$ cells from five mice). (c) Quantification of the mean distance to vasculature within contact, proximal and distal regions. ${ }^{\star \star \star \star} p<0.0001$ ( $n=43$ cells from five mice) by Student's $t$-test. (e) Quantification showing the incidence of Msi2GFpbright cells localized in contact with, proximal or distal to endosteum ( $n=33$ cells from five mice). (f) Quantification of the mean distance to endosteum within contact, proximal and distal regions. ${ }^{\star \star \star} P=0.0003$ ( $n=33$ cells from five mice) by Student's t-test. (g) Representative image of E15.5 Msi2GFP fetal liver showing Msi2GFPbright cells (green) localized near vasculature (white). Scale bar, $40 \mu \mathrm{m}$. (h) Quantification showing the incidence of Msi2GFpbright cells localized in contact with, proximal or distal to vasculature. (i) Quantification of the mean distance to vasculature within contact, proximal and distal regions. ${ }^{\star \star \star \star} P<0.0001$ ( $n=52$ cells) by Student's $t$-test. Data represented as mean + s.e.m.

\section{Discussion}

The approach we report provides a new framework for integrating very high-resolution long-term in vivo imaging with a high-throughput computational analysis (Table 1). The ability to use confocal microscopy makes this approach to real-time imaging significantly more accessible and provides far greater multichannel resolution relative to existing two-photon in vivo microscopy ${ }^{15-16}$. This spatial clarity allowed visualization of fundamental biological processes such as cell migration, division, extravasation and intravasation. Imaging of both transplanted as well as endogenous haematopoietic cells from a Msi2GFP knock-in reporter mouse revealed that haematopoietic stem/ progenitor cells are generally localized in contact with the vasculature, but distally from bone. Finally, we report a tool that 


\begin{tabular}{|c|c|c|c|c|c|c|}
\hline & Microscope & $\begin{array}{l}\text { Microenvironment } \\
\text { labelling }\end{array}$ & Number of channels & Temporal resolution & Spatial resolution & $\begin{array}{l}\text { High-throughput } \\
\text { computational } \\
\text { analysis }\end{array}$ \\
\hline $\begin{array}{l}\text { Highlights } \\
\text { of imaging } \\
\text { strategy }\end{array}$ & $\begin{array}{l}\text { Leica SP5 II } \\
\text { confocal }\end{array}$ & $\begin{array}{l}\text { - Genetic promoter } \\
\text { driven: knock-in } \\
\text { Msi2 reporter } \\
\text { (REM2) } \\
\text { Transgenic Notch } \\
\text { reporter } \\
\text { - Genetic-Backlit } \\
\text { microenvironment: } \\
\text { actin-dsRed, CFP } \\
\text { and GFP } \\
\text { - In vivo probe } \\
\text { injection } \\
\text { - In vivo antibody } \\
\text { injection } \\
\text { - Vascular dye } \\
\text { injection }\end{array}$ & 4 & Hours (up to $12 \mathrm{~h}$ ) & $\begin{array}{l}\text { - Can detect cell-cell } \\
\text { interactions. } \\
\text { - Can detect donor- } \\
\text { host cell interactions. } \\
\text { - Can detect cell } \\
\text { rolling and } \\
\text { extravasation. } \\
\text { - Can detect cell } \\
\text { divisions and } \\
\text { apoptosis. } \\
\text { - Can detect the } \\
\text { microenvironment via } \\
\text { antibody labelling } \\
\text { in vivo. }\end{array}$ & $\begin{array}{l}\text { - Can perform } \\
\text { quantitiative single- } \\
\text { cell analysis providing } \\
\text { detailed } \\
\text { spatiotemporal } \\
\text { information on } \\
\text { position, motion and } \\
\text { proximity to any niche } \\
\text { cell. } \\
\text { - Enables rapid and } \\
\text { efficient analysis of } \\
\text { multiple parameters } \\
\text { in vivo decreasing } \\
\text { manual input time by } \\
\text { 100-fold. }\end{array}$ \\
\hline $\begin{array}{l}\text { Advantages } \\
\text { over other } \\
\text { the systems }\end{array}$ & $\begin{array}{l}\text { Commercially } \\
\text { available, } \\
\text { widely } \\
\text { accessible and } \\
\text { economic } \\
\text { system }\end{array}$ & $\begin{array}{l}\text { - Backlighting and } \\
\text { reporters allow the } \\
\text { observation of } \\
\text { multiple cell types } \\
\text { in the } \\
\text { microenvironment } \\
\text { with the } \\
\text { transplanted donor } \\
\text { cells. } \\
\text { - Microenviron- } \\
\text { mental cells can be } \\
\text { visualized clearly } \\
\text { during long-term } \\
\text { imaging at single- } \\
\text { cell resolution. } \\
\text { - Msi2 reporter } \\
\text { allows imaging of } \\
\text { endogenous stem/ } \\
\text { progenitor cells. }\end{array}$ & $\begin{array}{l}\text { - Allows concurrent } \\
\text { visualization of } \\
\text { multiple cell types, } \\
\text { thereby enabling } \\
\text { mapping of } \\
\text { interactions of } \\
\text { haematopoietic cells } \\
\text { with distinct elements } \\
\text { of the niche. }\end{array}$ & $\begin{array}{l}\text { - Allows distinction } \\
\text { between transient } \\
\text { (seconds) and } \\
\text { stable (up to } 12 \mathrm{~h} \text { ) } \\
\text { interactions of } \\
\text { haematopoietic cells } \\
\text { with elements in the } \\
\text { microenvironment. }\end{array}$ & $\begin{array}{l}\text { - Allows tracking of a } \\
\text { wide range of cellular } \\
\text { behaviour in vivo } \\
\text { including asymmetric } \\
\text { cell division and } \\
\text { analysis of fine } \\
\text { interactions in the } \\
\text { microenvironment. }\end{array}$ & $\begin{array}{l}\text { - Provides a solution } \\
\text { to the current } \\
\text { difficulty in processing } \\
\text { 'big data': large } \\
\text { volumes of visual } \\
\text { information derived } \\
\text { from imaging. } \\
\text { - Analysis can } \\
\text { precisely discern novel } \\
\text { biological patterns of } \\
\text { cellular dynamics } \\
\text { in vivo } \\
\text { - Approach can be } \\
\text { broadly applied to } \\
\text { studying cellular } \\
\text { dynamics of other } \\
\text { tissues (for example, } \\
\text { gut) and other } \\
\text { processes (for } \\
\text { example, regeneration } \\
\text { and oncogenesis). }\end{array}$ \\
\hline
\end{tabular}

enables high-throughput computational analysis of the 'big data' generated from in vivo imaging. To our knowledge, a method to reliably automate spatiotemporal information from large volumes of imaging data does not exist at the current time; thus, the publicly available MATLAB program we have developed could be widely applicable, and thus a critical and novel contribution to the field.

The combination of the imaging resolution with the computational capacity provided, for the first time, quantified information about the activity of single cells in space and time in vivo. We analysed two microenvironmental elements implicated in haematopoiesis ${ }^{2-4,6-12}$ and show that immature haematopoietic cells appear to interact with each in distinct ways, with preferential contact made with vascular domains, and equivalent levels of proximal associations made with vascular and endosteal domains. In the long term, overlaying the base spatiotemporal map with a map of molecular reporter activity may allow definition of the signals that are differentially activated in response to particular cell-niche associations. Our strategy could be further expanded to study the dynamics of cell responses to injury, oncogenesis or inflammation. The microenvironmental changes in each of these contexts will be unique and need to be tracked; for example, after injury such as chemotherapy or radiation massive degeneration of microenvironmental structures occurs (Supplementary Fig. 5), indicating that associations may change markedly in regeneration. The temporal resolution achieved could be useful in gaining insight into the dynamics of haematopoietic cells in biological processes such as regeneration that unfold over time.

Analysing large amounts of image data has become a critical bottleneck in the discovery process ${ }^{26}$. To resolve this, we developed software that allows efficient quantitative characterization of each cell in terms of its position, motion and proximity to important niches. Approximately 41,968 frames were analysed in $20 \mathrm{~min}$ and required a total manual input time of $\sim 3.5 \mathrm{~h}$. To compare it with fully manual analysis, we estimated that a person would need at least $30-60 \mathrm{~s}$ per frame to measure the distance between a cell and each microenvironmental domain, and a total of $350-700 \mathrm{~h}$, indicating the software decreased manual work by 100 -fold. The fact that the software provides a marked advantage over manual processing suggests it can serve as a more general high-throughput tool for spatiotemporal analysis of in vivo imaging data. The software also allowed calculation of the velocity of blood cells moving both within the bone marrow or flowing through a blood vessel. This capability could be useful, for example, in defining how closely 'induced' blood cells 
generated from directed differentiation strategies resemble 'naturally born' blood cells. In fact as the field of regenerative medicine matures, it is intriguing to speculate that this type of tool could prove important for in vivo assessment of cells derived from directed differentiation methods before clinical use $\mathrm{e}^{27}$.

Our imaging analysis identified both spatial and temporal differences in the interactions of haematopoietic cells with vascular and endosteal regions in homeostasis (Supplementary Movie 10). Further, it revealed that the dynamic behaviour of haematopoietic cells in vivo is directly related to their differentiation state. Thus, haematopoietic stem/progenitor cell-enriched fractions have far higher spatial and temporal affinity for vascular domains, whereas differentiated cells shuttle more frequently between the vascular and endosteal domains, and exhibit less stable interactions. This suggests that immature cells are more dependent on the niches they are part of and thus retained more readily, and that this dependence diminishes with maturation, allowing cells to leave. In the longer term, the introduction of additional niche markers as well as other cell types could easily be accommodated by these techniques and allow the development of a comprehensive dynamic atlas of haematopoietic cell interactions within the bone marrow microenvironment.

The Msi2GFP reporter mouse is an exciting tool that has enabled us to dynamically track endogenous immature haematopoietic cells both temporally and spatially. Haematopoietic stem/progenitor cells in Msi2 reporter mice were largely localized in contact with the vasculature, and distally from the endosteum, suggesting that the vascular niche is particularly supportive of these undifferentiated cells in this context as well. Interestingly, the association of Msi2GFP bright cells with the vascular domain was highly stable, with almost all encounters scored as 'long' interactions. The dominance of stable long interactions was in contrast to the more temporally distributed interactions (long and short) recorded from transplanted stem/progenitor cells. While we have used the Msi2 reporters to establish a baseline for normal haematopoietic stem and progenitor cells, they could be useful in multiple contexts: for example, we have used this model to track heterogeneity within aggressive solid cancers, and find it enables successful identification of tumour propagating cells, and therapy resistance in pancreatic cancer ${ }^{18}$.

The work reported here highlights the power of visualizing tissues using high-resolution live microscopy to illuminate the bone marrow environment that is critical for the self-renewal and differentiation of haematopoietic stem and progenitor cells. The ability to observe different cell types simultaneously in vivo is a powerful tool for analysing and understanding the nature of processes such as regeneration or oncogenesis, where new regulators may be difficult to discover with static approaches alone. Because the principles of the imaging paradigm and computational analysis we developed can be applied broadly, our work also raises the exciting possibility that the use of this strategy may ultimately allow a dynamic view into an array of tissues and organs whose architecture and living physiology will be important areas of future investigation.

\section{Methods}

Animals and cell isolation. Haematopoietic stem and progenitor cells were isolated from 8-12-week-old Actin-GFP mice (Jackson Labs, Bar Harbor, ME, USA) as described ${ }^{28}$. Whole bone marrow was lineage depleted via magnetic-activated cell sorting (MACS; Miltenyi Biotec, Bergisch Gladbach, Germany) using an AutoMACS sorter (Miltenyi Biotec). Subsequent lineagedepleted cells were stained using phycoerythrin (PE)-conjugated antibodies CD3e, CD4, CD8, B220, CD11b, Gr-1 and Ter119 (eBiosciences), and sorted for Lin ${ }^{+}$ and Lin ${ }^{-}$fractions. Analysis and cell sorting were carried out on a FACSVantage sorter (Becton Dickinson, Mountain View, CA, USA) at the Duke Cancer Center Flow Cytometry Core Facility. A total of $1.5 \times 10^{6} \mathrm{GFP}^{+} \mathrm{Lin}^{-}$progenitors were transplanted via retro-orbital sinus into p15 DsRed2 or 8-week-old mice (STOCK $\operatorname{Tg}\left(\right.$ CAG-DsRed ${ }^{*}$ MST $) 1$ Nagy/J, Jackson Labs). Mice were imaged between 1 and $12 \mathrm{~h}$ after adoptive transfer of $\mathrm{GFP}^{+} \mathrm{Lin}^{+}$and $\mathrm{GFP}^{+} \mathrm{Lin}^{-}$progenitors. For KLS cell isolation, whole bone marrow was enriched for c-Kit via MACS (Miltenyi Biotec) using an AutoMACS (Miltenyi Biotec). c-Kit-enriched cells were labelled for PE-conjugated antibodies for CD3e, CD4, CD8, B220, CD11b, Gr-1 and Ter119, APC-conjugated antibody c-Kit, and PE-Cy5-conjugated antibody for Sca-1 (eBiosciences). Analysis and cell sorting were carried out on a FACS AriaIII sorter (Becton Dickinson). A total of $1.5 \times 10^{6} \mathrm{GFP}^{+} \mathrm{KLS}$ cells were transplanted via retro-orbital sinus into 8 -week-old mice (STOCK Tg(CAG-DsRed ${ }^{\star}$ MST) 1Nagy/J, Jackson Labs). Mice were imaged $24 \mathrm{~h}$ after adoptive transfer of $\mathrm{GFP}^{+}$KLS cells. REM2 (Msi2 ${ }^{+/ G F P}$ ) reporter mice were generated by conventional gene targeting (Genoway, France). The eGFP cassette was knocked into exon 1 of the Msi2 gene in frame with the ATG start codon ${ }^{18}$. Msi2GFP reporter mice imaged were between 3 and 8 weeks of age. Both male and female mice were used for the experimental purposes. All animal experiments were performed according to the protocols approved by the Duke University and University of California, San Diego, Institutional Animal Care and Use Committee.

Mouse preparation and imaging. Mice were anaesthetized by intraperitoneal injection of ketamine and xylazine $\left(100 / 20 \mathrm{mg} \mathrm{kg}^{-1}\right)$. Once mice were unresponsive to pedal reflex, heads were wiped down with $70 \%$ ethanol and hair was removed using Nair Hair Remover lotion (Church \& Dwight Co., Inc., Princeton, NJ, USA). A midline incision was made using FST ToughCut Spring Scissors, 6-mm curved blade (Fine Science Tools Inc., Foster City, CA, USA), and the skin was removed to expose the calvarium. For inverted confocal microscopy using younger mice, the calvarium was kept moist with Aqua Poly/Mount (Polysciences, Inc., Warrington, PA, USA) during the imaging session. Mice were inverted and secured onto a custom microscope rig by placing a rubber band (size 10) through the bit of the mouth and observed through a $22 \times 22-\mathrm{mm}$ coverslip (VWR International, West Chester, PA, USA). Mice were immediately taken to the confocal microscope for imaging and were kept under anaesthesia using $1-3 \%$ isofluorane gas mixed with oxygen. For upright confocal microscopy, mice were placed in a mouse/neonatal rat stereotactic holder (Stoelting Co., Wood Dale, IL, USA), calvarium was exposed as described above and tissue was kept moist using $1 \times$ PBS (Gibco).

Microscopy. Images were acquired by Leica LAS AF 1.8.2 software with either an inverted Leica SP5 confocal system using a Leica DMI6000CS microscope or an upright Leica SP5 2 confocal system using a Leica DM 6,000 CFS microscope. Using the inverted microscope, images were acquired using a $\times 10$ Leica Plan Apochromat objective with 0.40 numerical aperture for quantification and a $\times 20$ Leica Plan Apochromat objective with 0.70 numerical aperture. Using the upright microscope, images were acquired using an HCX APO L20x objective with a 1.0 numerical aperture for still images and subsequent movies. Imaging of calvarium ranged from 60 to $100 \mu \mathrm{m}$. CFP (excitation $458 \mathrm{~nm}$ and emission $463-500 \mathrm{~nm}$ ), GFP (excitation $488 \mathrm{~nm}$, emission 493-556 nm) and DsRed2 (excitation $561 \mathrm{~nm}$, emission 566-650 nm) were excited with an Argon/2 (458, 477, 488, 496 and $514 \mathrm{~nm})$ and Diode pumped solid-state $(561 \mathrm{~nm})$ laser, respectively. The power used for dsRed visualization was $8-12 \%$ of the appropriate laser. Images were continuously captured in $1,024 \times 1,024$ or $1,024 \times 512$ format, with line averaging of 4 ( $\sim 10$ or $5 \mathrm{~s}$ per scan, respectively) for up to $8 \mathrm{~h}$. Multicolour imaging for CFP and GFP were captured sequentially.

Methylcellulose colony formation assay. LT-HSCs (KLSCD $150^{+} \mathrm{CD} 48^{-}$) were isolated by FACS from bone marrow. Cells were plated in methylcellulose medium (Methocult GF M3434 from StemCell Technologies). Colonies were counted 7 days after plating.

In vivo transplantation assay. A total of 500 LT-HSCs isolated from bone marrow of mice expressing CD45.2 were transplanted into lethally irradiated (9.5 Gy) CD45.1 recipient mice with $3 \times 10^{5}$ Sca-1-depleted CD45.1 bone marrow cells. Peripheral blood of recipient mice was collected at 4 weeks after transplantation.

Chemotaxis assay. Directed cell migration towards SDF1 was analysed in vitro. Cells were kept in X-VIVO media (Lonza) and $600 \mu \mathrm{l} X$-VIVO media supplemented with $50 \mathrm{ng} \mathrm{ml}^{-1}$ of SDF1 was added to the lower chamber of the transwell (Costar, pore size $5 \mu \mathrm{m}, 3,421$ ). A total of 75,000 cells were loaded into the upper chamber and allowed to migrate for $18 \mathrm{~h}$ at $37^{\circ} \mathrm{C}$ in a humidified $\mathrm{CO}_{2}$ incubator. After incubation, migrated cells were collected from the lower chamber and counted. 
Ex vivo fetal liver preparation and microscopy. Mouse embryonic fetal livers were dissected at stage E15.5 from timed mating females. Fetal livers were incubated on ice with fluorescently conjugated antibodies for VE-Cadherin (eBiosciences). Fetal livers were plated for imaging in $1.5 \%$ low melting agarose (Sigma) with $X$-VIVO media (Lonza) and $10 \%$ fetal bovine serum. Cultures were maintained at $37^{\circ} \mathrm{C}$ and $5 \% \mathrm{CO}_{2}$ using a Heating Insert P Lab-Tek S1 with an Incubator PM S1 (Zeiss). Images were acquired using an Axio Observer Z1 microscope with the LSM 700 scanning module (Zeiss).

In vivo probe administration. Angiosense 680 and OsteoSense 680 in vivo imaging probes (VisEn, Bedford, MA, USA) were administered at a concentration of $2 \mathrm{nM}$ in $150 \mu \mathrm{l}$ per mouse, and imaged within $30 \mathrm{~min}$ (Angiosense) or at least $24 \mathrm{~h}$ (OsteoSense) after administration. Both products were excited using a $\mathrm{HeNe}$ 633 laser and emission was collected from 650 to $725 \mathrm{~nm}$. Antibodies conjugated to AlexaFluor 647 for VE-cadherin (eBiosciences) and F4/80 (eBiosciences) were administered at a concentration of $10 \mu \mathrm{g}$ diluted in $100 \mu \mathrm{l}, 15 \mathrm{~min}$ before imaging. All products were excited using a $\mathrm{HeNe} 633$ laser and emission was collected from 650 to $725 \mathrm{~nm}$.

Quantitative analysis. Images were analysed using Volocity Software (Improvision, a PerkinElmer Company, Coventry, England). Red and green channel noise was optimized using the fine filter, and image intensity gamma was used to reduce background within the green channel. For GFP quantification, $\times 10 z$-stacks ( $30 z$-planes for $120 \mu \mathrm{m}$ ) were analysed. Briefly, objects were filtered by intensity and size, and the sum of the isovolume $\left(\mu \mathrm{m}^{3}\right)$ measurements was compared between the mice. Image enhancement and quantification parameters were identical between paired animals for each experiment. Movies were made using Volocity software and exported to view as AVI movies at 15 frames per second, and compressed using Microsoft Video 1 compression. Supplementary Movie 2 rolling movie frame rate was reduced to 5 frames per second.

High-throughput imaging analysis. All image processing and object tracking were performed in MATLAB (R2010b). Supplementary Movie 11 is a representative movie that was used for the analysis. First, the movies were corrected for lateral $(x y)$ drift by examining the cross-correlation between the first frame and every subsequent frame. Images labelled with cells were median filtered with a window size of 5 pixels, and then thresholded with a manual cutoff. Each candidate cell object was identified and the centroid calculated in each frame. Cells were tracked through time using particle-tracking software (http://physics.georgetown.edu/matlab/) and only tracks longer than 10 frames $(\sim 100 \mathrm{~s})$ were considered valid. The cells moved occasionally in three dimensions, briefly disappearing from the image for certain time points. In such cases, the position data for that time point was estimated by linear interpolation between the values obtained from the preceding and following images. As a final check, the quantified data ( $x, y$ positions for each cell at each time point-as shown in Supplementary Data 1) was superimposed on the image stacks, and the resulting movies were then subjected to a manual review to ensure that no errors were made in tracking. To classify cell locations as either vascular or endosteal, cell positions were compared with hand annotated images of vascular and endosteal regions, as shown in Supplementary Fig. 6. The minimum distance to a vascular and endosteal region was calculated for each position. To specify proximal and contact interactions, the distances to vascular and endosteal regions were compared across all data sets for all time points and distance cutoffs chosen appropriately. Interactions were classified as vascular or endosteal based on which region was closest, and contact and proximal interactions were decided with the previously described distance cutoffs. False interactions were suppressed in two ways: (1) contact and proximal distances were automatically adjusted by $\pm 1 \mu \mathrm{m}$ for each cell and (2) adjacent transient interactions $(<200 \mathrm{~s})$ were merged together. Finally, the track and interaction graph for each cell was verified by manual inspection. MATLAB scripts and extracted data files have been provided as Supplementary Software.

Data availability. The data that support the findings of this study are available from the corresponding author on request.

\section{References}

1. Orkin, S. H. \& Zon, L. I. Hematopoiesis: an evolving paradigm for stem cell biology. Cell 132, 631-644 (2008).

2. Yin, T. \& Li, L. The stem cell niches in bone. J. Clin. Invest. 116, 1195-1201 (2006).

3. Kiel, M. J. \& Morrison, S. J. Uncertainty in the niches that maintain haematopoietic stem cells. Nat. Rev. Immunol. 8, 290-301 (2008).

4. Wilson, A. \& Trumpp, A. Bone-marrow haematopoietic-stem-cell niches. Nat. Rev. Immunol. 6, 93-106 (2006).

5. Wagers, A. J. The stem cell niche in regenerative medicine. Cell Stem Cell 10, 362-369 (2012).

6. Arai, F. et al. Tie2/angiopoietin-1 signaling regulates hematopoietic stem cell quiescence in the bone marrow niche. Cell 118, 149-161 (2004).
7. Kiel, M. J. et al. SLAM family receptors distinguish hematopoietic stem and progenitor cells and reveal endothelial niches for stem cells. Cell 121, 1109-1121 (2005).

8. Zhang, J. et al. Identification of the haematopoietic stem cell niche and control of the niche size. Nature 425, 836-841 (2003).

9. Calvi, L. M. et al. Osteoblastic cells regulate the haematopoietic stem cell niche. Nature 425, 841-846 (2003).

10. Kunisaki, Y. et al. Arteriolar niches maintain haematopoietic stem cell quiescence. Nature 502, 637-643 (2013).

11. Nombela-Arrieta, C. et al. Quantitative imaging of haematopoietic stem and progenitor cell localization and hypoxic status in the bone marrow microenvironment. Nat. Cell Biol. 15, 533-543 (2013).

12. Butler, J. M. et al. Endothelial cells are essential for the self-renewal and repopulation of Notch-dependent hematopoietic stem cells. Cell Stem Cell 6, 251-264 (2010).

13. Acar, M. et al. Deep imaging of bone marrow shows non-dividing stem cells are mainly perisinusoidal. Nature 526, 126-130 (2015).

14. Sipkins, D. A. et al. In vivo imaging of specialized bone marrow endothelial microdomains for tumor engraftment. Nature. 435, 969-973 (2005).

15. Lo Celso, C. et al. Live-animal tracking of individual haematopoietc stem/progenitor cells in their niche. Nature 457, 92-96 (2009).

16. Lo Celso, C. et al. In vivo imaging of transplanted hematopoietic stem and progenitor cells in mouse calvarium bone marrow. Nat. Protoc. 6, 1-14 (2011).

17. Kohler, A. et al. Altered cellular dynamics and endosteal location of aged early hematopoietic progenitor cells revealed by time-lapse intravital imaging in long bones. Blood 114, 290-298 (2009).

18. Fox et al. Image based detection and targeting of therapy resistance in pancreatic adenocarcinoma. Nature 534, 407-411 (2016).

19. Ito, T. et al. Regulation of myeloid leukaemia by the cell-fate determinant Musashi. Nature 466, 765-768 (2010).

20. Mazo, I. B. et al. Hematopoietic progenitor cell rolling in bone marrow microvessels: parallel contributions by endothelial selectins and vascular cell adhesion molecule 1. J. Exp. Med. 188, 465-474 (1998).

21. Vintersten, K. et al. Mouse in red: red fluorescent protein expression in mouse ES cells, embryos, and adult animals. Genesis 40, 241-246 (2004).

22. Bevis, B. J. \& Glick, B. S. Rapidly maturing variants of the Discosoma red fluorescent protein (DsRed). Nat. Biotechnol. 20, 83-87 (2002).

23. Mizutani, K., Yoon, K., Dang, L., Tokunaga, A. \& Gaiano, N. Differential Notch signalling distinguishes neural stem cells from intermediate progenitors. Nature 449, 351-355 (2007).

24. Duncan, A. W. et al. Integration of Notch and Wnt signaling in hematopoietic stem cell maintenance. Nat. Immunol. 6, 314-322 (2005).

25. Wu, M. et al. Imaging hematopoietic precursor division in real time. Cell Stem Cell 1, 541-554 (2007).

26. Walter, T. et al. Visualization of image data from cells to organisms. Nat. Methods 7, S26-S41 (2010).

27. Vo, L. T. \& Daley, G. Q. De novo generation of HSCs from somatic and pluripotent stem cell sources. Blood 125, 2641-2648 (2015).

28. Domen, J. The role of apoptosis in regulating hematopoiesis and hematopoietic stem cells. Immunol. Res. 22, 83-94 (2000).

\section{Acknowledgements}

We are grateful to Larry Katz and Ben Arenkiel for critical experimental advice on live animal imaging and microscopy. We also thank members of the Reya lab for suggestions and help during the development of this imaging system; Brigid Hogan, Tim Oliver and Sam Johnson for advice regarding microscopy and image analysis; Anthony Means, Brigid Hogan and Nelson Chao for comments on the manuscript; and Michael Cook and Beth Harvat for cell sorting. T.R. is the recipient of a Leukaemia and Lymphoma Society Scholar Award, C.S.K. was supported by National Institutes of Health (NIH) training grant T32 GM007752 (UCSD), J.R.H. was supported by NIH training grant EB01630 (Duke University) and U19 pilot award AI067798, B.Z. was supported by T32 GM007184-33 (Duke University), T.I. is the recipient of a California Institute for Regenerative Medicine interdisciplinary stem cell training program fellowship, and T.K.L. was supported by a Stanford Graduate Fellowship and NIH training grant 5T32GM008412. R.G.F. is a recipient of a California Institute for Regenerative Medicine interdisciplinary stem cell training program fellowship and also received support from NIH training grants T32 HL086344 and T32 CA009523 (UCSD). This work was also supported by NIH grants CA125994, DP1OD006413, as well as a Hellman Faculty Scholarship to M.W.C. and NIH grants DK072234, AI067798, HL09776, DP1 CA174422, R35 CA197699 and an Alexander and Margaret Stewart Fund grant to T.R.

\section{Author contributions}

C.S.K. and J.R.H. designed and performed the majority of experiments and helped write the manuscript. J.R.H. and S-H.J developed the live mouse imaging system. J.W., R.G.F., B.Z., T.I., A.B. and J.P.C. provided experimental support, advice and help. A.C. developed 
the image projection. T.K.L. and M.W.C. developed the computational analysis software, performed quantitative analysis of real-time images and helped write the manuscript. T.R. conceived of the project, planned and guided the research, and wrote the manuscript.

\section{Additional information}

Supplementary Information accompanies this paper at http://www.nature.com/ naturecommunications

Competing financial interests: The authors declare no competing financial interests.

Reprints and permission information is available online at http://npg.nature.com/ reprintsandpermissions/
How to cite this article: Koechlein, C. S. et al. High-resolution imaging and computational analysis of haematopoietic cell dynamics in vivo. Nat. Commun. 7:12169 doi: $10.1038 /$ ncomms12169 (2016).

cc (i) This work is licensed under a Creative Commons Attribution 4.0 International License. The images or other third party material in this article are included in the article's Creative Commons license, unless indicated otherwise in the credit line; if the material is not included under the Creative Commons license, users will need to obtain permission from the license holder to reproduce the material. To view a copy of this license, visit http://creativecommons.org/licenses/by/4.0/

(C) The Author(s) 2016 